\title{
Impact Visualisation in Educational Interventions
}

\author{
ZhiMin Xiao ${ }^{\mathrm{a}}$, Steve Higgins ${ }^{\mathrm{b}}$, and Adetayo Kasim ${ }^{\mathrm{c}}$ \\ ${ }^{a}$ Graduate School of Education, University of Exeter, Exeter, DH1 2LU, UK \\ ${ }^{\mathrm{b}}$ School of Education, Durham University, Durham, DH1 1TA, UK \\ ${ }^{\mathrm{c}}$ Wolfson Research Institute, Durham University, Stockton Campus, Stockton-on-Tees, TS17 6BH, UK
}

\section{ARTICLE HISTORY}

Compiled November 20, 2018

\begin{abstract}
Reporting of research data analysis often resorts to numerical summaries, such as effect size estimates in Randomised Controlled Trials (RCTs). Summary statistics are helpful and important for evidence synthesis and decision making. However, they can be unstable and inconsistent due to diversity in research designs and variability in analytical specifications. They also mask the dynamics of individual responses to a certain intervention by focusing on average treatment effect on the treated, even though the variation in impact may be crucial information for policy makers. To establish stability and consistency of impact estimates and to reveal the dynamics of individual responses in RCTs, we conduct variable selection, harness the power of noise, implement Cumulative Quantile Analysis (CQA), and devise umbrella plots of loss and gain in this study, using real datasets from over 30 educational interventions funded by the Education Endowment Foundation (EEF) in England. For the purpose of comparison, which is essential in data visualisation, all the aforementioned methods are built upon multiple analytical approaches. We show that the importance of an intervention can be ordered through variable selection, and that the power of noise or the bias induced by inappropriate variables, can be utilised to assess the stability of an impact estimate. We also demonstrate that estimates of average treatment effect cannot fully capture the impact of an intervention on sub-groups of participants with varying levels of attainment at baseline, not to mention individual responses to the intervention. Using CQA and umbrella plots, we are able to supplement what common effect size estimates lack in educational interventions. We argue that the impact of an intervention is often more complex than the average treatment effect suggests, and that until a summary is more informative and able to speak directly to the eye, evidence-based policy and practice cannot be fully achieved.
\end{abstract}

\section{KEYWORDS}

Effect size; Evidence; Impact; Noise; RCT; Visualisation

CONTACT Z. Xiao. Email: z.m.xiao@exeter.ac.uk 


\section{Introduction}

Research scientists usually employ numerical summaries, such as effect sizes, to estimate the impact of social interventions. Like all statistical indexes, effect sizes are not perfect measures, as they focus on average treatment effect. If an educational intervention has a large impact on higher attaining pupils but a small one on lower attaining pupils, the average treatment effect would be negligible because the gain is cancelled out by approximately the same amount of loss. An effect size estimate from such a study is therefore misleading, as it risks preventing a large proportion of students from benefiting from the intervention, should the small effect size suggest the study not be scaled up. Also, if an intervention has more or less the same impact on high and low attaining pupils, but very different effects on boys and girls, the effect size estimate may be a sufficient measure of impact for all the pupils involved in the study, but once gender is statistically controlled for, the estimate can change in sign and/or magnitude, which is a case of Simpson's Paradox (Wainer and Brown 2004, 2006; Lord 1967, 1969; Tu, Gunnell, and Gilthorpe 2008), a statistical phenomenon that has puzzled statisticians for decades (Holland 1986).

Moreover, numerical summaries mask the dynamics of individual responses to a specific intervention in a given study. When mixed methods are employed in the evaluation of the intervention, the findings from numerical estimations often contradict with the lived experiences of individual participants. This is not uncommon, for instance, in some of the EEF studies (Jerrim et al. 2016; Haywood et al. 2015), the headline effect sizes were small, but reportedly, participants were largely enthusiastic about the interventions. Also, teachers and process evaluators of the studies observed positive changes amongst the students. Such mismatch of quantitative and qualitative findings adds to the challenge of evidence-based policy. Nevertheless, numerical summaries such as effect size estimates are still common and unavoidable until new analytical methods are invented and widely adopted. Also, they are unlikely to disappear soon because the public often demand straightforward answers, even though research evidence is more often than not messy and qualitative and quantitative findings often collide. While it is possible that a social intervention produces different types of evidence that point to the same direction, it is in fact very rare, at least in many of the EEF trials. Clearly, numerical summaries are unable to fully capture the complexity of research evidence. As consumers of research findings, we should not expect them to meet the expectations of all. 
That said, we should bear in mind that numerical summaries have significantly contributed to changes, be they positive or negative, in the society we live in (Malakoff 2017 ; O'Neil 2016). Statistically speaking, they are useful for evidence synthesis across multiple studies of a similar phenomenon, for instance, health impact of smoking (Brandt 2009). It is the very numerical summaries that have led to the ban of public smoking. In education, academic performance is always condensed into similar summaries from primary schooling to advanced studies, almost everywhere. Even in academia, numeric metrics are employed to assess academic impact, allocate resources amongst educational institutions, reward or punish individual academics. In finance, similar summaries determine who is credit worthy. All in all, effect size estimates can and have contributed to the promotion of public goods when they are well used, but at the same time, they do mask the diversity of individual preferences, mute cultural sensitivity, and sometimes aggregate structural inequalities when they are misused or abused. That is to say, numerical summaries underpin how modern societies function and have consequences for nearly all aspects of our daily lives. We cannot live without them, whether we like or loathe them.

To minimise the negative consequences of numerical summaries, progressive approaches that embrace the complex nature of social interventions are always in need. In this study, we introduce three graphical methods that promise to improve or at least improve the communication of numerical summaries. First, we treat all variables in a given dataset as signals and noises. Unlike most analysts, we do not prefer the former to the latter. Instead, we employ several detecting mechanisms to pick up the signals when multiple variables are viewed as a challenge or even a 'curse'. Meanwhile, we harness the power of noise, in which case, multiple variables are regarded as a blessing. In either case, we show that it is important to plot multiple numerical summaries derived from several analytical models in a single plot for a given study, as the visualisation approach allows us see how stable numerical summaries are across different models of analysis. If summaries generated by multiple models are stable, we have more confidence in the results. Otherwise, further explorations are needed to explain the variation due to analytical choices. After all, without comparison, 'numbers on their own are not enough' (Gelman and Unwin 2013 p. 5) and graphics have to build on careful data analyses (Tukey 1993). In other words, graphics and analyses supplement each other, a grabbing graphic may be worth a thousand words, but an arresting image plus a thousand words can achieve even more (Friendly 2008), as together they invite readers to intellectually involve with the results visualised (Gelman and Unwin 2013). 
Second, following the visualisation that focuses on stability, we investigate the consistency of intervention effect using a Cumulative Quantile Analysis (CQA), which divides the data from an educational trial into four additive quantiles according to individual performances at baseline before applying an analytical model of choice to the first up to all the quantiles considered. If the effect size estimates from the CQA remain consistent across all the quantiles, we can make a more robust conclusion than otherwise that the intervention has an even impact on all the participants, regardless of their academic capabilities at baseline. However, if the estimates from the CQA vary considerably from one quantile to another, it indicates that the intervention benefits only a subset of the samples selected for the study. Note that the patterns of estimates across different quantiles can be consistent, increase, decrease, or fluctuate as more and more participants are included for analysis. When it increases, it means higher performing pupils gained more from an intervention than lower performing pupils did, which suggests a Matthew Effect. When it decreases, it indicates the intervention had a greater impact on lower performing pupils, who might be the target of the intervention. When it fluctuates, a definitive conclusion cannot be drawn given the data.

Finally, we devise an umbrella plot to visualise the loss and gain of individuals in the intervention and control groups of an intervention. As the name of the plot suggests, if the intervention has no impact at all on an outcome of interest, the loss and gain distributions for the two groups should resemble each other: the proportions of pupils who gained or lost from pre- to post-test should decrease as the expected amount of gain or loss increases. In other words, most observations would cluster around the no gain or loss vertical line of zero, as most pupils would not gain or lose by a substantial amount in a typical educational intervention. It should be noted that this is the relative difference between the groups and aims to identify the difference in progress between the two groups. The 'loss' is therefore relative and pupils classified this way may still have made progress from the pre-test. However, if the intervention has a large effect in boosting pupil performances from baseline to post-test, the difference-in-proportions of pupils who gained or lost by a certain amount would favour those in the intervention group, that is to say, fewer pupils in the intervention group than those in the control group would lose relatively across different levels of loss in the loss side of the plot, but more of the former than the latter would gain across different levels of gain in the gain side of the plot. In all cases, the loss (negative) and gain (positive) is calculated as the difference between pre- and post-test scores, which are standardised before the former is subtracted from the latter. The vertical line 
of zero represents no gain or loss, relative to the overall distribution. To the right or left of the line from zero to positive or negative one on the $x$-axis are varying levels of gain or loss, with a difference at each level in the proportions of pupils in the intervention and control groups who gained or lost on the $y$-axis. In the vertical direction, we also plot all the observations in either intervention or control group for all levels of gain or loss.

\section{Too Many Variables! A Curse or Blessing?}

Research datasets, such as those from EEF trials listed in Table1, usually come with many variables. Trial data can be cleaner as a result of successful randomisation. However, randomisation does not guarantee balance on all covariates (Torgerson and Torgerson 2008), which prompts researchers of educational interventions to collect data on more variables than pre-test measures and intervention group status. In EEF evaluations, different evaluation teams tend to control for different covariates in their final analytical models. Sometimes, effect size estimates are simply a difference-in-means of post-tests between intervention and control groups; other times, a range of covariates, including possible interactions and higher order terms, are introduced. When the estimates of effect vary considerably as a result of such choices, the presence of multiple covariates is thus a curse, as it adds to the uncertainty of effect size estimation. However, when systematic approaches are employed to choose signal or weed out noise variables, the end result from them should not vary too much. Common approaches in linear regression include forward selection and backward elimination of covariates. Occasionally, an exhaustive search of the best subsets of covariates at a given number is implemented (James et al. 2015), thanks to increasing computational power.

In this section, we first treat multiple covariates as a 'curse', which requires us to detect signals. To accomplish the goal, we employ the three variable selection methods mentioned above to conduct noise reduction for some of the EEF datasets examined. We expect the three methods to end up with similar but slightly different selections. Other analysts of research data may use any of the three methods to find the best model for effect size estimation, we use all three at the same time to assess the importance of the covariates considered, particularly intervention group status variable called $t$ in the EEF studies. Prior to the selection process, we re-organise the data in such a way that the first variable is the outcome, which is followed by other covariates according to their strength of correlation with the outcome. That is to say, 
the second variable or column in the dataset has the largest correlation coefficient with the first one or the outcome. The last variable or column has the weakest correlation with the first one.

To reflect sampling uncertainty, we also bootstrap the observed sample 100 times, and in each iteration, the same three variable selection methods are implemented. In the end, we count how many times each of the covariates in a dataset is selected for each of the three selection methods. If a covariate is picked up 100 times, we code the cells in Figures 1 and 2 as dark blue. As we can see, it is rare to have such a covariate when a model has very few covariates, such as one, two, or three. However, as the model grows in complexity in terms of covariate number, more covariates are likely to be selected 100 times, just as all the cells in the bottom rows are dark blue in the figures, because all models in the study have an intercept. Since the covariates are ordered according to their correlation coefficients with the outcome, the order of covariates in the Figures roughly represents the order of importance, with the most important ones plotted as foundation variables in each of the three sub-figures for either of the two real datasets illustrated. If we focus on the covariate $t$, its importance differs in the two studies. When an intervention has a large effect, as in Figure 1 for Improving Writing Quality, the covariate gets selected early by any of the three selection methods. When an intervention has no effect, as in Figure 2 for Chess in Schools, the covariate appears near the top of the $y$-axis. We also note that, the three selection methods, in the long run, choose about the same covariates for models with the same number of covariates, or of similar complexity. For instance, when the number of covariates is two, covariate $t$ in Figure 1 is picked up $55 \%, 62 \%$, and $53 \%$ of the time by exhaustive, backward, and forward selection methods respectively. If we do not bootstrap the data 100 times, the models with two covariates can be very different across the three methods. While pret is most certain to appear, gender is likely to replace t $44 \%, 32 \%$, and $45 \%$ of the time across the three selection methods.

Covariates, including their higher order and interaction terms, can also be viewed as a blessing when we harness the power of undesirable variables or noise in analytical models, because they introduce a source of bias to the estimation of an intervention effect. Depending on the strength of signal in a given dataset, that bias varies in power. A common practice in data analysis is to discard, as much as possible, the influence of such noise, or to reduce the bias in estimation. If an effect size estimate in an educational intervention can withstand the maximum power of noise, we can be more certain of the intervention effect than otherwise. To maximise the magnitude of bias in a given dataset, we include all linear and quadratic terms, which are second order and 
interaction terms of all observed covariates in the data. As shown in Figure 3 , the total number of covariates varies from intervention to intervention, as the number of observed variables differs from study to study. In all four cases shown, we apply to the same dataset a series of simple linear regression models controlling for different number of covariates, which increases from one, namely, intervention group status indicator, to all possible pairwise combinations of linear and quadratic terms. As the model gets more and more complex, the magnitude of the bias introduced increases as well. The effect size estimates from all the models, as plotted in the $y$-axis, fluctuate as the number of covariates, as shown in the $x$-axis, changes.

To make the effect size estimates as comparable as possible across the four studies in Figure 3. we simply convert the primary outcome of each study into $z$-scores, which are then used as the outcome in the linear regression models described above. As a result, the coefficient on intervention group status is close to Hedges' $g$ (Hedges 2007), a metric widely used in the analysis of trial data (Borenstein 2009). For a given study, the range of effect estimates from all the models considered thus reflect the uncertainty associated with analytical choices. Note that the analytical uncertainty is different from the normal sampling uncertainty, which we also embrace in the visualisation when covariates are considered as noise. In Figure 3 , the orange squares represent point estimates based on the actual sample, upon which multiple linear regressions with varying number of covariates are fitted. Since every point estimate has an associated sampling distribution, we also bootstrap the actual sample 100 times, and each time, the same multiple linear regression models are fitted to the bootstrapped datasets, which result in 100 effect size estimates for each level of model complexity. We save all the estimates before plotting the median estimate together with the one from the actual sample. The bootstrapped effect size estimates from across the models with different number of covariates form a similar but slightly different distribution, which is plotted as green dots in Figure 3.

\section{Cumulative Quantile Analysis}

In the previous section, we devised a method to establish the stability of effect size estimates by selecting signal variables and harnessing the power of noise. In signal detection, if treatment indicator is consistently picked up early by the three variable selection methods, we know that the study has a large intervention effect. If it remains amongst the last ones to be chosen, we know that the intervention effect is small. Also, if an effect estimate stands more or less the same 
regardless of the power of noise, we are more certain of the effect estimate, as the range of effect size estimates, based on observed and simulated datasets, embraces sampling and analytical uncertainties. Nevertheless, those point estimates are still average treatment effects, suggesting they are unable to capture the variation of an intervention effect amongst the groups of pupils with varying capabilities at baseline. As pointed out earlier, one limit of averaging is the cancelout effect of gains and losses, which masks the information about the sub-groups of participants who actually gained or lost in an intervention. To overcome that problem, we need a progressive approach that can show us how consistent an intervention effect is across sub-groups of pupils with varying attainment levels at baseline. If an intervention effect is inconsistent across the subgroups, the average treatment effect would be misleading, as it cannot tell us which attainment groups actually benefitted most from the intervention.

To show the consistency of intervention effect across different attainment groups at baseline, we first divide the data from a given RCT into four quantiles based on pre-test scores. This approach would be most reliable when the sample size is large, but some EEF trials are relatively small, we therefore conduct a Cumulative Quantile Analysis, where the samples in the first quantile are included in the analysis of the second quantile, the first two quantiles included in the analysis of the third quantile, until the fourth quantile analysis where all of the sample is used. As the sample size increases from one quantile to the following one, the uncertainties surrounding the effect estimates should be narrower and narrower. If an intervention has a similar point estimate across the four quantiles, the study must have been well implemented and targeted the correct groups of pupils. But it also indicates that most participants in the study find the intervention equally beneficial or ineffective, depending on whether the effect size estimate is positive or negative.

Figure 4 shows 50 sets of effect size estimates from 31 educational interventions funded by the EEF (some interventions have multiple outcomes, hence more outcomes than intervention projects). Each set of estimates comprises three point estimates and their associated uncertainties at $95 \%$ level. From left to right, the three vertical bars represent results from three different analytical models, which are difference-in-means, multilevel modelling (using total variance), and ordinary least squares. As we can see, the estimates from the three models can differ substantially, as in, for instance, efm in (a), shine and ttsm in (c). If we focus on the results from one model each time and compare them across the studies, we can also see that point estimates of effect can be consistent, fluctuate, increase, or decrease from the first quantile to 
the fourth. In general, uncertainties surrounding those point estimates decrease as we include more samples. For those studies that have consistent point estimates from across the quantiles, such as ar in sub-figure (a), mms in (b), p4cm in (c), and impn in (d), we have more confidence in the estimates and conclude that the studies targeted the correct groups of pupils. When the point estimates decline from lower to higher quantiles, as in bp, catchn, and rti in (a), shine in (c), and aspm in (d), we conclude that the interventions had a greater impact on lower performing pupils than they had on higher performing ones. Likewise, if the point estimates increase from the first to the fourth quantile, as in sar and text in (b), ttpe in (c), and quest in (d), we view it as an indication that higher performing pupils in those studies benefited more from the interventions than lower performing pupils did. If we look at point estimates from multilevel modelling alone, there are 19 out of the 50 outcomes that have point estimates above zero across all four quantiles. 11 out of the 50 outcomes have point estimates below zero across the quantiles, which indicate that the interventions had negative impact on the participants in terms of performance on post-test. Other outcomes fluctuate or remain at the zero horizontal line. Taken together, the results suggest that by and large, the EEF interventions had positive impacts on pupil attainment.

\section{Umbrella Plot of Loss and Gain}

So far, we have demonstrated how average treatment effect withstands noise in the data and how consistent effect size estimates are across different quantiles, but neither method reveals how individuals respond to a certain intervention. In this section, we deploy a new visualisation method called umbrella plot, which shows how many pupils in intervention and control groups gained or lost from baseline to post-test and by how much. When an intervention has a large effect, there will be more pupils in the treatment group than in the control group who gain from baseline to post-test. Take Improving Writing Quality in Figure 5 for example, $20 \%$ more pupils in the treatment group gained by at least 0.1 standard deviation from pre- to post-test, $16 \%$ more gained by at least 0.2 standard deviation. As the amount of gain increases, the proportion of pupils, as represented by the $y$-axis of the umbrella plot, in either intervention or control group decreases. However, in this particular study (a), the difference-in-proportions remains substantial across all levels of gain. If we focus on loss in the left-hand side of sub-figure (a), we can find the same story: fewer pupils in the intervention group than in the control group lost 
across all levels of loss from 0 to -1 , and the difference-in-proportions is similar to that in the gain side of the plot.

Figure 5 (a) actually shows two distinct umbrellas rather than one. This is quite different from the other three sub-figures of umbrella plot, where two umbrellas almost overlap. In other words, the distance between the red and blue lines is larger in the former than in the latter, which suggests the former had a larger intervention effect than the latter. So ideally, we want to see two umbrellas placed in two unique places, with the red line below (or above) the blue one in the loss (or gain) side of the two umbrellas. Any deviation from the pattern suggests a different or no intervention effect. For instance, Chess in Schools (c) has two umbrellas almost overlapping each other, suggesting a small, if any, overall intervention effect. However, the red line in the loss side stands above the blue line between 0 and -0.6 , meaning more pupils in the treatment group actually lost up to 0.5 standard deviation from baseline to post-test, relative to the mean of both groups. If we look at the gain side of the plot, we can see that fewer pupils in the treatment group than those in the control group gained across all levels of gain. This indicates that some pupils in Chess in Schools made greater overall progress than the control group, but that this was counterbalanced by those who did not progress as well. The intervention seems to have widened the distribution of those taught to play chess.

ReflectEd (d) is another interesting plot. The average treatment effect is almost negligible, which means the intervention had a low intervention effect if we look at the headline effect size estimate alone (Motteram et al. 2016). However, when we move from the numerical summary of effect size to the umbrella plot, we see a slightly different picture. Although the intervention did not help more pupils in the treatment group gain from the intervention, it was quite effective in preventing more pupils from relative loss. In other words, at almost all levels of loss, fewer pupils in the intervention group than those in the control group lost. This suggests the intervention narrowed the spread, which is consistent with meta-cognitive interventions having greater impact on low attaining pupils. This pattern should be encouraged, as most interventions focus on gain rather than loss. When gain is hard to achieve amongst certain groups of pupils, success in the prevention of loss should be equally desirable, if not more than gain.

Since all umbrella plots in Figure 5 show all observations at all levels of loss and gain, the length of the vertical bars gives us an indication of sample sizes in a specific study. For instance, Improving Writing Quality has longer vertical bars at all levels of loss and gain than the other three, meaning the former has a smaller sample size than the other three. Longer bars thus 
represent greater uncertainties surrounding the point estimates of an intervention effect. We can imagine these distributions of individual responses as rain drops, when a distribution is long in the vertical direction, it is analogous to torrential rain, which blocks visibility, suggesting instability of effect size estimates. When the weather is fair, we do not see much rain, the distributions are shorter in the vertical direction, suggesting the results are more stable than in rainy conditions.

\section{Conclusion}

The visualisation methods we have introduced in the study can expose different snapshots of an intervention effect. Unlike numerical summaries, they let us see the stability of point estimates from multiple analytical models at the same time. While conventional methods use metrics such as a $p$-value to indicate whether an observed difference between intervention and control groups is likely to be random or not, we have demonstrated how important an intervention is, relative to other measures in the data, in making a difference in outcome between the groups concerned. We also plot out as many effect estimates as possible, given the data we have access to, to gain a better idea about the range of possible point estimates for a given study. This analytical uncertainty is different from conventional sampling uncertainty under the frequentist framework of inference, which is usually derived from a mathematical theory rather than real data. While the stability of effect estimates relates to the choices researchers make on research design, data processing and analytical methods, the consistency of an intervention effect, as reflected in the results of CQA, concerns the intrinsic function of an intervention itself. The patterns of point estimates in CQA would paint a similar picture about an intervention to that encoded in a numerical summary of effect size, if the intervention targeted the correct group of participants, was consistently implemented across different quantiles, or had an equal impact on all pupils included. As the EEF datasets explored in this study show, the ideal scenario of consistency across all the quantiles is not always the case. The patterns can in fact fluctuate, increase, or decrease, which suggests numerical summaries can be incomplete at best, or misleading at worst. Umbrella plots of individual responses to an intervention illustrate this point even further. For instance, when an intervention is effective in preventing many students from loss, it may be desirable, even if an average treatment effect suggests otherwise. Moreover, umbrella plots remind researchers and evaluators of the importance of focusing on both loss and gain, rather 
than gain alone, as is the case in many social interventions.

While the visualisation methods we have shown in the study are more informative than conventional numerical summaries, they are meant to supplement rather than substitute them. They can show evaluators and decision makers how an intervention worked, down to individual level if necessary, and offer 'productive possibilities' (McCosker and Wilken 2014) when they are viewed as 'door openers' (Gelman and Unwin 2013) or the 'means for generating understanding' (McCosker and Wilken 2014) rather than the end of statistical analyses. But they are not yet ready for evidence synthesis, where aggregated information from multiple studies of a similar phenomenon is necessary for policy decisions. Given current development in data visualisation, a graphical method that addresses the issue may be possible in the near future. However, this is beyond the scope of this study. Besides, the methods introduced here share some of the limitations of numerical summaries. For example, they both rely upon the validity and reliability of pre- and post-tests. For this reason, even when the visualisation methods and numerical summaries are employed at the same time, it is still important to consult qualitative data gathered from process evaluations, where stakeholder attitudes, experiences, and opinions are solicited. Another limitation of the visualisation approach is that it cannot address some concerns regarding RCTs, for instance, the control or business-as-usual groups we see in many EEF studies are not, arguably, the ideal controls we often see in the experimental designs of other fields than social sciences. After all, a 'Magic Breakfast' intervention in schools can never have a control group where pupils are not allowed to eat their breakfast at home. Given the limitations listed above, we join many others in stressing the importance of diversifying research methods for evidence, just as we abandon our reliance on a single best model in the visualisation methods shown in the study.

\section{Disclosure statement}

We have no conflicts of interest to disclose.

\section{Funding}

This research was funded by a grant to Durham University from the Education Endowment Foundation in England. 


\section{Acknowledgement}

We acknowledge the vision of the Education Endowment Foundation in creating a data archive of their educational trials, which will enable further exploration and development; and we thank the staff at FFT Education Ltd for their data curation of this archive. We would also like to acknowledge our thanks to Drs. Andrey Sivachenko and Victor Farutin, who inspired the first author to implement some of the concepts and techniques in R.

\section{References}

Biggart, Andy. 2015. "Quest." London. https://goo.gl/arpLGP.

Borenstein, Michael. 2009. "Effect Sizes for Continuous Data." In The handbook of research synthesis and meta-analysis, edited by Harris Cooper, Larry V. Hedges, and Jefferey C. Valentine, 2nd ed., Chap. 12, 221-235. New York: Russell Sage Foundation.

Brandt, Allan M. 2009. The Cigarette Century: The Rise, Fall, and Deadly Persistence of the Product That Defined America: A Cultural History of Smoking in the United States. New York: Basic Books. Buchanan, Emily, Jo Morrison, Matthew Walker, Helen Aston, and Rose Cook. 2015. "Tutor Trust Secondary." London. https://goo.gl/cEe4SG

Friendly, Michael. 2008. "The Golden Age of Statistical Graphics." Statistical Science 23 (4): 502-535. Gelman, Andrew, and Antony Unwin. 2013. "Infovis and Statistical Graphics: Different Goals, Different Looks." Journal of Computational and Graphical Statistics 22 (1): 2-28.

Gorard, Stephen, Beng Huat See, and Nadia Siddiqui. 2014. "Switch-on Reading." London. https: //goo.gl/qAvzxM.

Gorard, Stephen, Nadia Siddiqui, and Beng Huat See. 2014a. "Effective Feedback." London. https: //goo.gl/mTbNL3.

Gorard, Stephen, Nadia Siddiqui, and Beng Huat See. 2014b. "Future Foundations." London. https: //goo.gl/rQYkXx.

Gorard, Stephen, Nadia Siddiqui, and Beng Huat See. 2014c. "Response to Intervention." London. https: //goo.gl/D1DUY5.

Gorard, Stephen, Nadia Siddiqui, and Beng Huat See. 2015a. "Accelerated Reader." London. https: //goo.gl/aCSdQj.

Gorard, Stephen, Nadia Siddiqui, and Beng Huat See. 2015b. "Fresh start." London. https://goo.gl/ aYiEnN

Gorard, Stephen, Nadia Siddiqui, and Huat See. 2015c. "Philosophy for Children." London. https: 
//goo.gl/D8tcPF.

Haywood, Sarah, Julia Griggs, Cheryl Lloyd, Stephen Morris, Zsolt Kiss, and Amy Skipp. 2015. "Act, Sing, Play." London. https://goo.gl/CNLFeq

Hedges, Larry V. 2007. "Effect Sizes in Cluster-Randomized Designs." Journal of Educational and Behavioral Statistics 32 (4): 341-370.

Holland, Paul W. 1986. "Statistics and Causal Inference." Journal of the American Statistical Association 81 (396): 945-960.

James, Gareth, Daniela Witten, Trevor Hastie, and Robert Tibshirani. 2015. An Introduction to Statistical Learning: with Applications in R. 6th ed., Vol. 64. New York: Springer.

Jerrim, John, Helen Austerberry, Cosette Crisan, Anne Ingold, Candia Morgan, Dave Pratt, Cathy Smith, and Meg Wiggins. 2015. "Mathematics Mastery." London. https://goo.gl/QknZg7.

Jerrim, John, Lindsey Macmillan, John Micklewright, Mary Sawtell, and Meg Wiggins. 2016. "Chess in Schools." London. http://goo.gl/HSf1Gv.

King, Bernardine, and Adetayo Kasim. 2015. "Rapid Phonics." London. https://goo.gl/dpZrA8.

Lloyd, Cheryl, Triin Edovald, Zsolt Kiss, Stephen Morris, Amy Skipp, and Hashim Ahmed. 2015. "Paired Reading." London. https://goo.gl/NKQHvA.

Lord, Frederic M. 1967. "A paradox in the interpretation of group comparisons." Psychological Bulletin 68 (5): 304-305.

Lord, Frederic M. 1969. "Statistical adjustments when comparing preexisting Groups." Psychological Bulletin 72 (5): 336-337.

Lord, Pippa, Sally Bradshaw, Eleanor Stevens, and Ben Styles. 2015. "Perry Beeches Coaching Programme." London. https://goo.gl/4sdTP6.

Malakoff, David. 2017. "A Matter of Fact.” Science 355 (6325): 562-563.

Maxwell, Bronwen, Paul Connolly, Sean Demack, Liam O'Hare, Anna Stevens, and Lucy Clague. 2014a. "Summer Active Reading Programme." London. https://goo.gl/YTBNrC

Maxwell, Bronwen, Paul Connolly, Sean Demack, Liam O'Hare, Anna Stevens, and Lucy Clague. 2014b.

"TextNow Transition Programme." London. https://goo.gl/cV2bmp.

McCosker, Anthony, and Rowan Wilken. 2014. "Rethinking 'big data' as visual knowledge: the sublime and the diagrammatic in data visualisation." Visual Studies 29 (2): 155-164.

Menzies, Victoria, Clare Collyer, Dimitra Kokotsaki, Catherine Hewitt, Kirsty Younger, Andy Wiggins, and Carole Torgerson. 2015. "SHINE in Secondaries." London.

Merrell, Christine, and Adetayo Kasim. 2015. "Butterfly Phonics." London. https://goo.gl/KF3rxp.

Motteram, Gary, Sophina Choudry, Afroditi Kalambouka, Graeme Hutcheson, and Amanda Barton. 2016. "ReflectED." London. http://goo.gl/JX9oGX 
O’Neil, Cathy. 2016. Weapons of Math Destruction: How Big Data Increases Inequality and Threatens Democracy. New York: Crown Publishing Group.

Rienzo, Cinzia, Heather Rolfe, and David Wilkinson. 2015. "Changing Mindsets." London. https:// goo.gl/S9GVkF

Rutt, Simon. 2014. "Catch Up Numeracy." London. https://goo.gl/xHHxFi

Rutt, Simon. 2015. "Catch Up Literacy." London. https://goo.gl/ibejvP.

See, Beng Huat, Stephen Gorard, and Nadia Siddiqui. 2015. "Word and World Reading." London. https: //goo.gl/NT1KSi.

Sheard, Mary, Bette Chambers, and Louise Elliott. 2015. "Units of Sound." London. https://goo.gl/ rRzpEC,

Sibieta, Luke, Ellen Greaves, and Barbara Sianesi. 2014. "Increasing pupil motivation." London. https:

$$
\text { //goo.gl/xUxQ41. }
$$

Styles, Ben, and Sally Bradshaw. 2015. "Talk for Literacy." London. https://goo.gl/wvSAjJ.

Styles, Ben, Rebecca Clarkson, and Katherine Fowler. 2014a. "Chatterbooks." London. https://goo. gl/rgWS96

Styles, Ben, Rebecca Clarkson, and Katherine Fowler. 2014b. "Rhythm for Reading." London. https: //goo.gl/TSYzMW.

Styles, Ben, Eleanor Stevens, Sally Bradshaw, and Rebecca Clarkson. 2014. "Vocabulary Enrichment Intervention." London. https://goo.gl/p5EZVo.

Torgerson, David, Carole Torgerson, Hannah Ainsworth, Hannah Buckley, Clare Heaps, Catherine Hewitt, and Natasha Mitchell. 2014a. "Improving Writing Quality." London. http://goo.gl/YBuVsg.

Torgerson, David, Carole Torgerson, Natasha Mitchell, Hannah Buckley, Hannah Ainsworth, Clare Heaps, and Laura Jefferson. 2014b. "Grammar for Writing." London. https://goo.gl/BFjkXW.

Torgerson, David J, and Carole J Torgerson. 2008. Designing Randomised Trials in Health, Education and the Social Sciences: An Introduction. London: Palgrave Macmillan.

Tu, Yu-Kang, David Gunnell, and Mark S Gilthorpe. 2008. "Simpson's Paradox, Lord's Paradox, and Suppression Effects are the same phenomenon-the reversal paradox." Emerging themes in epidemiology 5: $1-9$.

Tukey, John W. 1993. "Graphic Comparisons of Several Linked Aspects: Alternatives and Suggested Principles." Journal of Computational and Graphical Statistics 2 (1): 1-33.

Wainer, Howard, and Lisa M Brown. 2004. "Two statistical paradoxes in the interpretation of group differences: Illustrated with medical school admission and licensing data." The American Statistician 58 (2): 117-123.

Wainer, Howard, and Lisa M. Brown. 2006. "Three Statistical Paradoxes in the Interpretation of Group 
Differences: Illustrated with Medical School Admission and Licensing Data." Handbook of Statistics 26 (06): 893-918.

Worth, Jack, Juliet Sizmur, and Ben Styles. 2015. "Improving Numeracy and Literacy." London. https : //goo.gl/9TcCLz. 

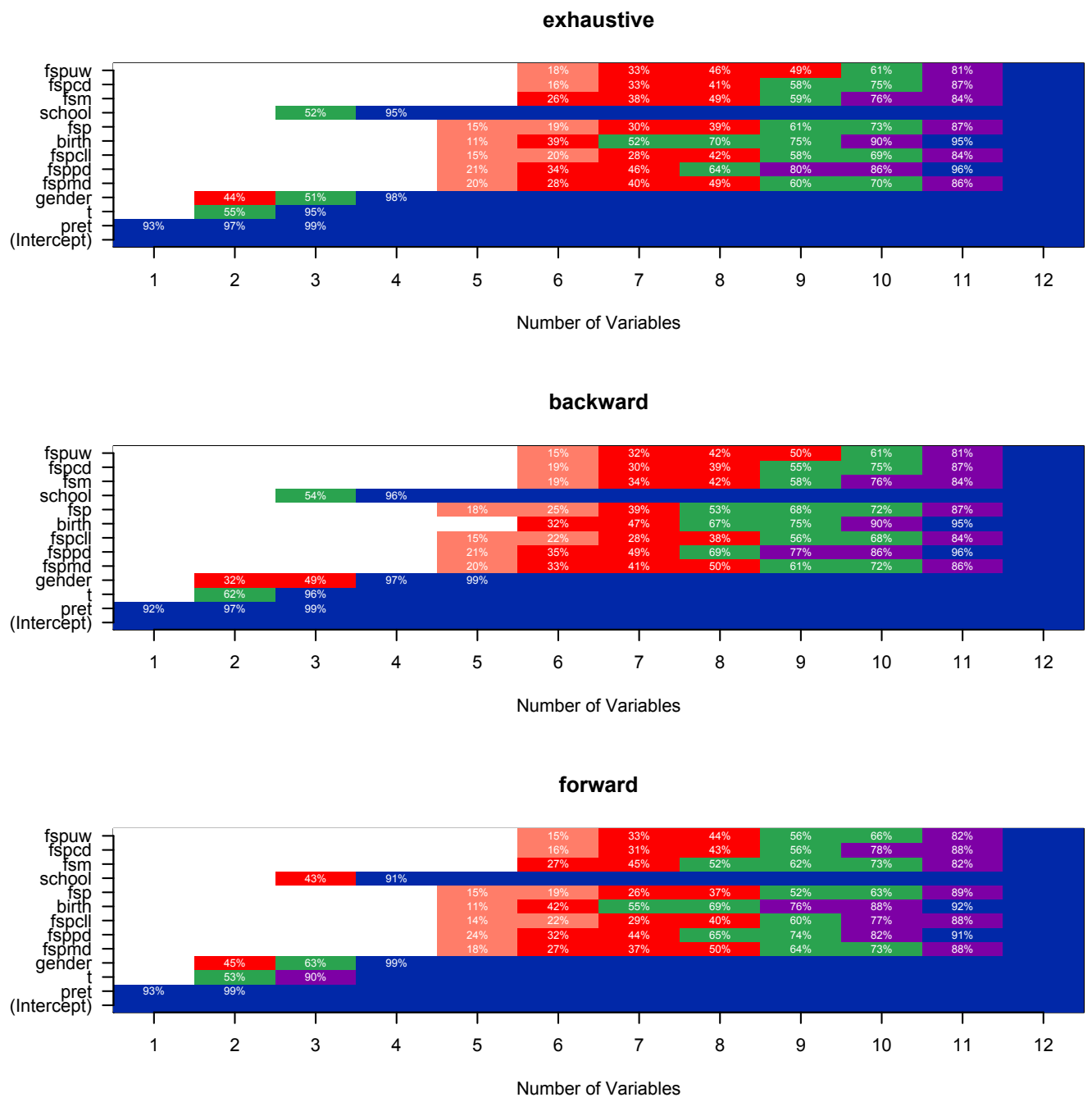

Figure 1.: Variable Selection for Improving Writing Quality: Three selection methods applied to the same datasets 100 times using bootstrapping. 


\section{exhaustive}

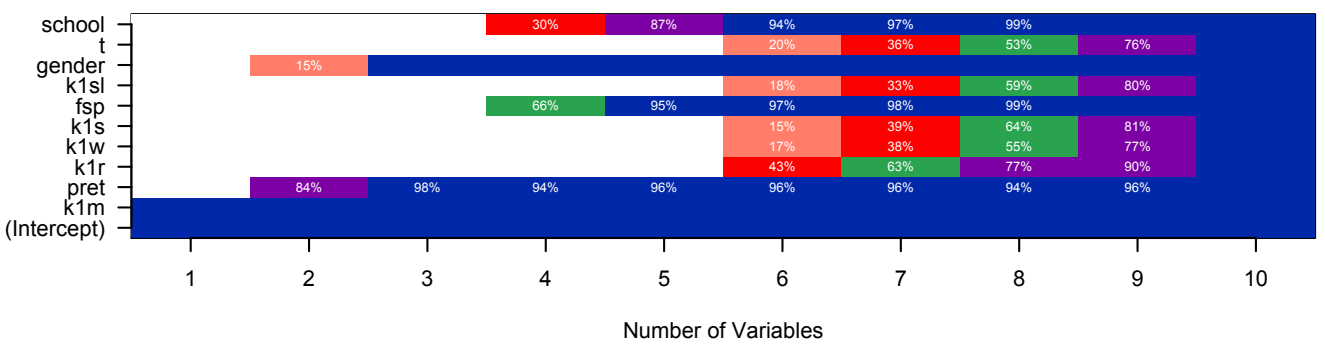

backward

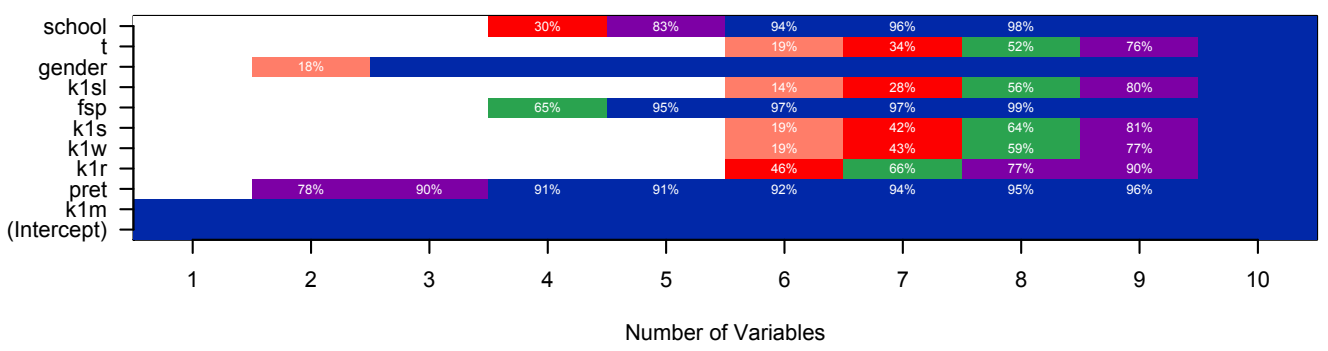

forward

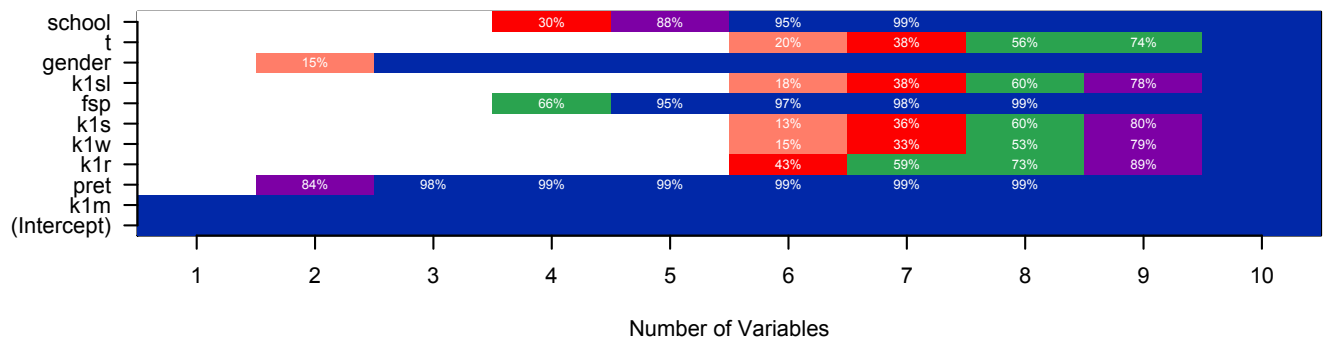

Figure 2.: Variable selection for Chess in Schools. 


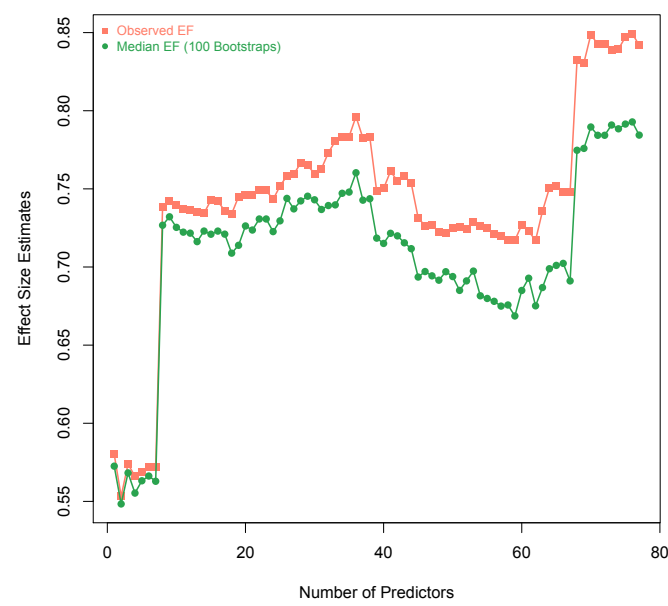

(a) Improving Writing Quality

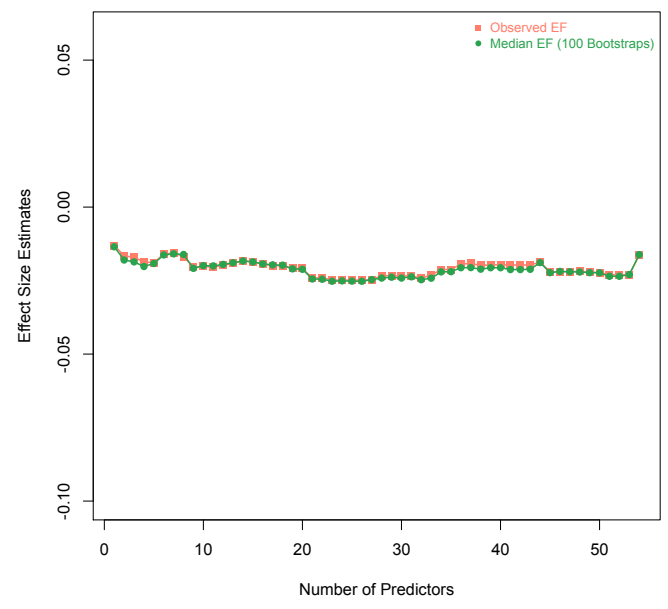

(c) Chess in Schools

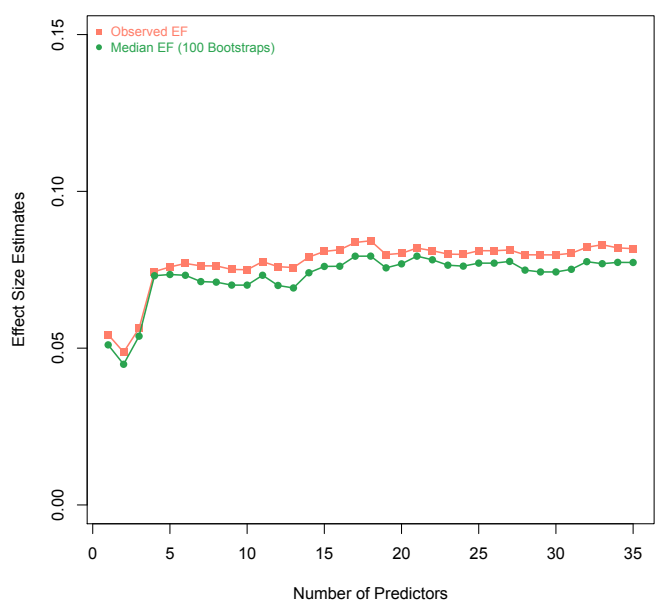

(b) Philosophy for Children

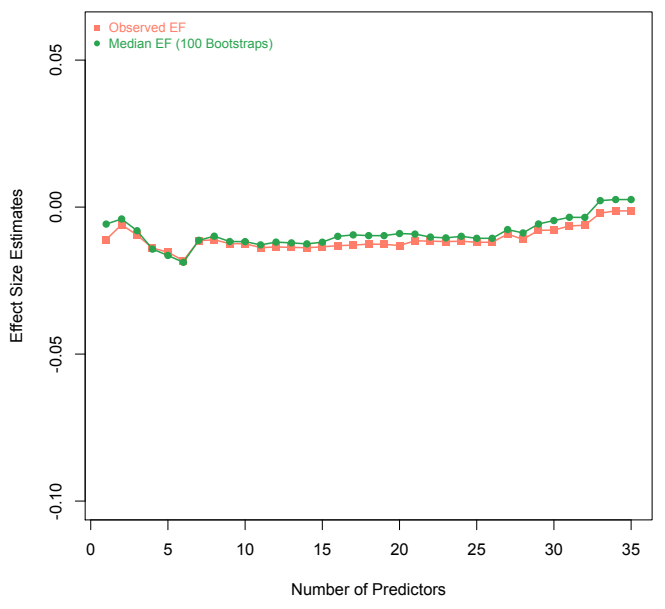

(d) ReflectEd

Figure 3.: Harnessing the power of noise to establish stability of effect size estimates using multiple models. 


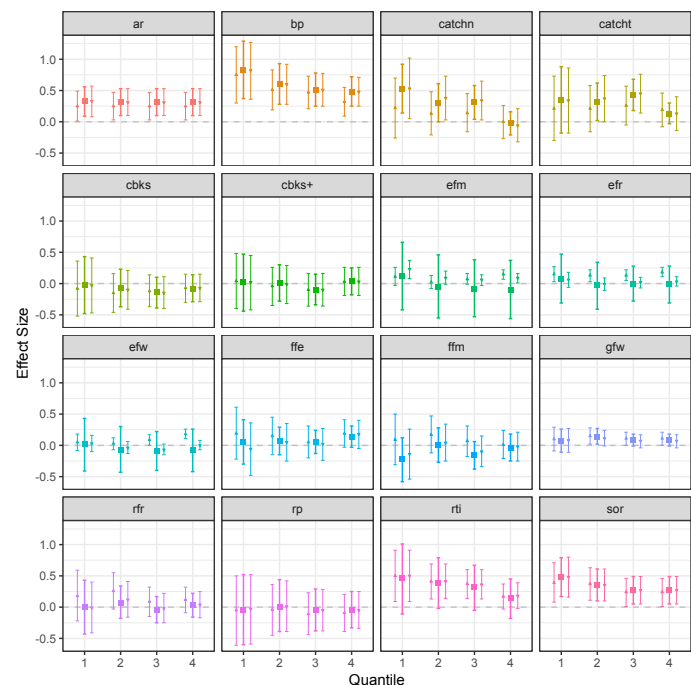

(a) First 16 Outcomes

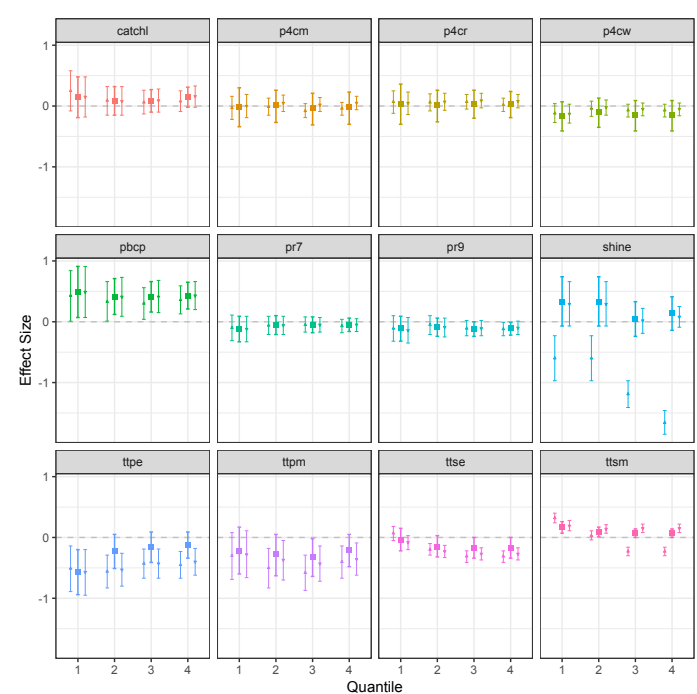

(c) Third 16 Outcomes

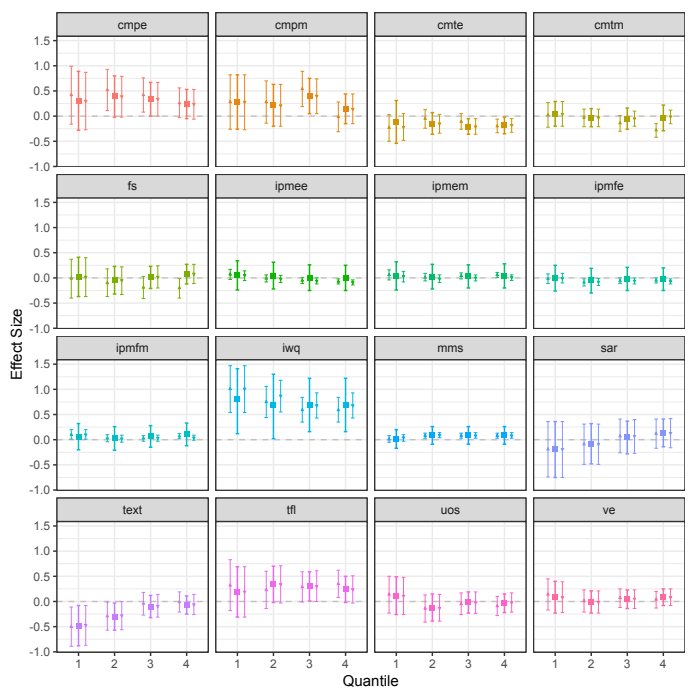

(b) Second 16 Outcomes

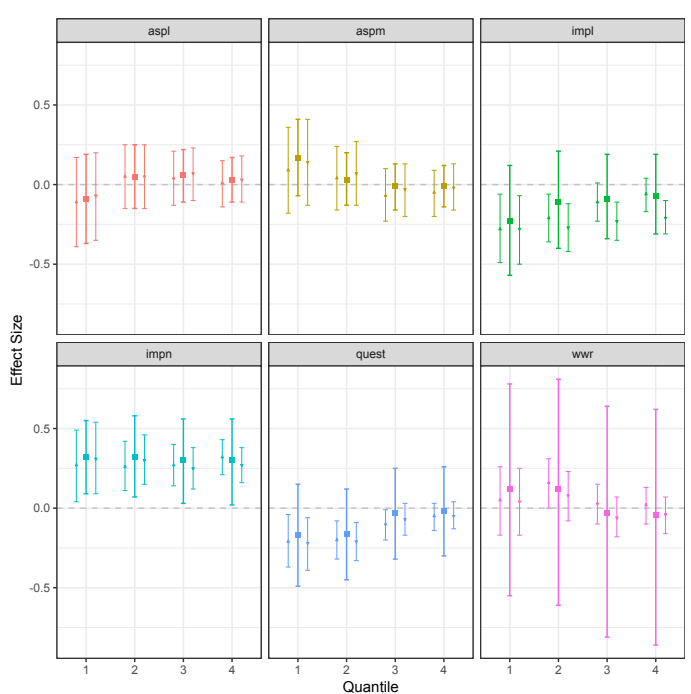

(d) Final 6 Outcomes

Figure 4.: Cumulative Quantile Analysis to test the consistency of point estimates from across the quantiles. From left to right, the three vertical bars represent results from Difference-inMeans, MLM (total), and OLS. 


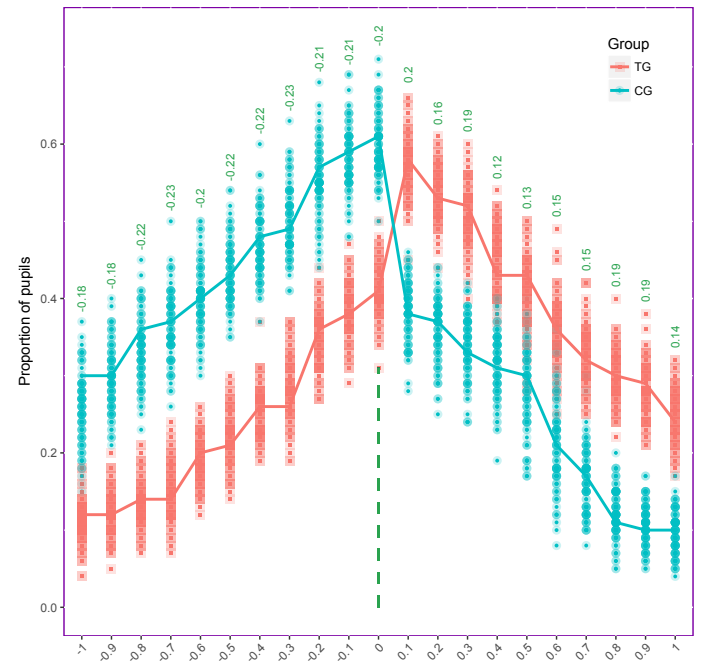

(a) Improving Writing Quality

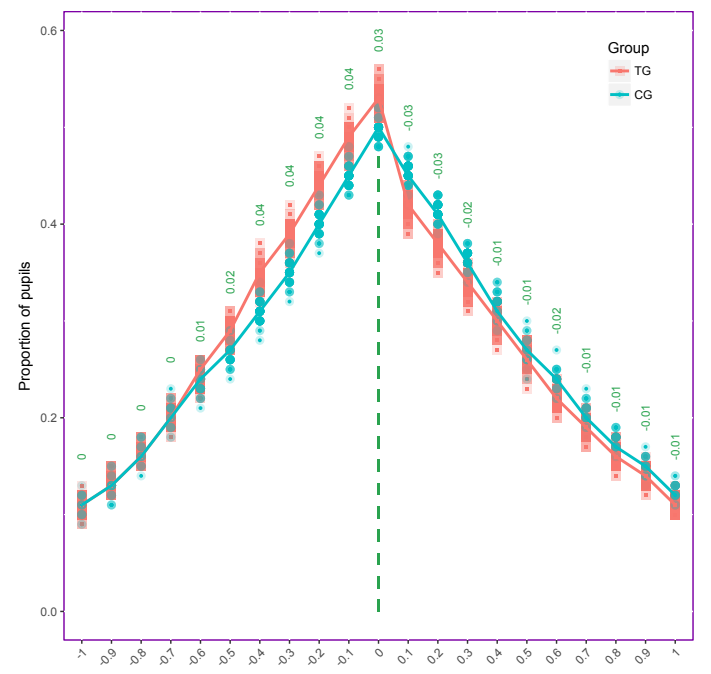

(c) Chess in Schools

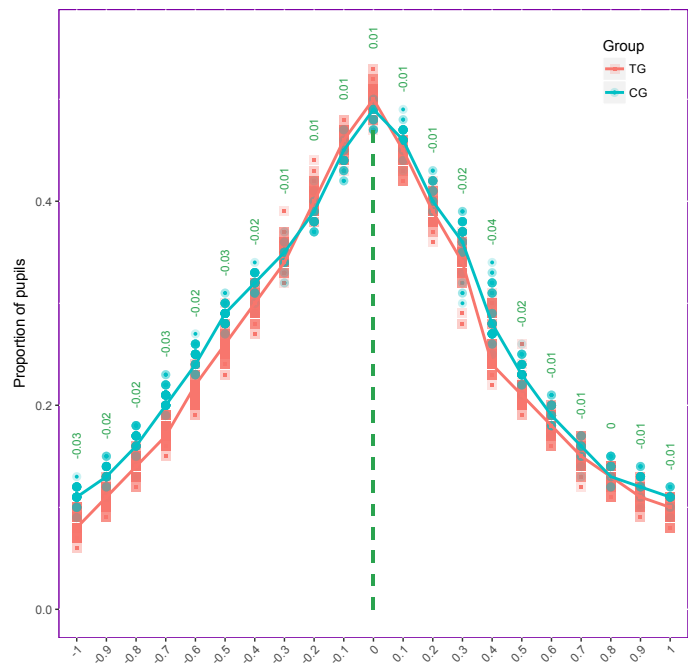

(b) Philosophy for Children

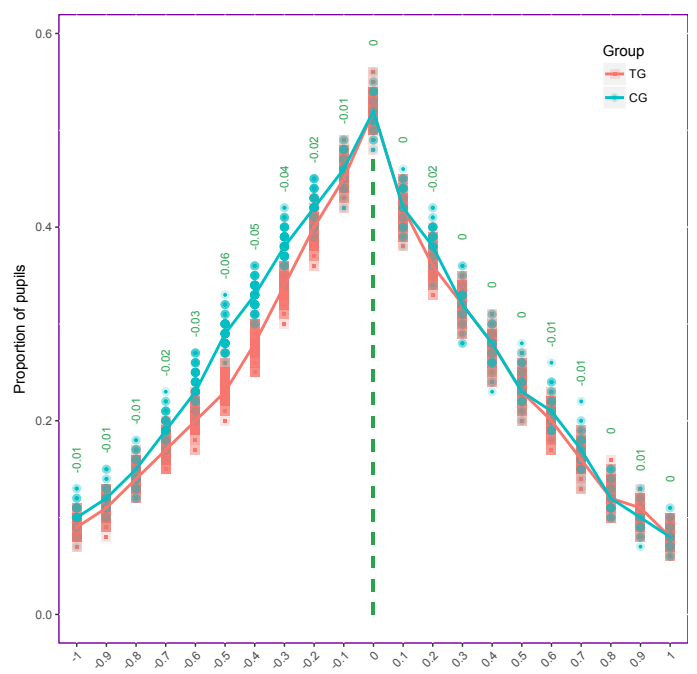

(d) ReflectEd

Figure 5.: Umbrella plots of individual responses to four educational interventions. 


\begin{tabular}{|c|c|c|}
\hline archive label & full EEF title & evaluation report \\
\hline ar & Accelerated Reader & $\begin{array}{l}\text { Gorard, Siddiqui, and } \\
\text { See }(2015 a)\end{array}$ \\
\hline bp & Butterfly Phonics & Merrell and Kasim \\
\hline catchn, catcht & Catch Up Numeracy & Rutt (2014) \\
\hline cbks, cbks+ & Chatterbooks & \begin{tabular}{|l|} 
Styles, Clarkson, and \\
Fowler $(2014 \mathrm{a})$
\end{tabular} \\
\hline efm, efr, efw & Effective Feedback & $\begin{array}{l}\text { Gorard, Siddiqui, and } \\
\text { See }(2014 a)\end{array}$ \\
\hline ffe, ffm & Future Foundations & $\begin{array}{l}\text { Gorard, Siddiqui, and } \\
\text { See }(2014 b)\end{array}$ \\
\hline gfw & Grammar for Writing & $\begin{array}{l}\text { Torgerson et al. } \\
(2014 b)\end{array}$ \\
\hline $\mathrm{rfr}$ & Rhythm for Reading & \begin{tabular}{|l|} 
Styles, Clarkson, and \\
Fowler $(2014 \mathrm{~b})$
\end{tabular} \\
\hline rp & Rapid Phonics & $\begin{array}{l}\text { King and Kasim } \\
(2015)\end{array}$ \\
\hline rti & Response to Intervention & $\begin{array}{l}\text { Gorard, Siddiqui, and } \\
\text { See }(2014 c)\end{array}$ \\
\hline sor & Switch-on Reading & $\begin{array}{l}\text { Gorard, See, and Sid- } \\
\text { diqui }(2014)\end{array}$ \\
\hline cmpe, cmpm, cmte, cmtm & Changing Mindsets & \begin{tabular}{|l|} 
Rienzo, Rolfe, and \\
Wilkinson $(2015)$
\end{tabular} \\
\hline fs & Fresh Start & $\begin{array}{l}\text { Gorard, Siddiqui, and } \\
\text { See }(2015 b)\end{array}$ \\
\hline ipmee, ipmem, ipmfe, ipmfm & Increasing Pupil Motivation & $\begin{array}{l}\text { Sibieta, Greaves, and } \\
\text { Sianesi }(2014)\end{array}$ \\
\hline iwq & Improving Writing Quality & $\begin{array}{l}\text { Torgerson et al. } \\
(2014 \mathrm{a})\end{array}$ \\
\hline mms & Mathematics Mastery Secondary & Jerrim et al. $(2015)$ \\
\hline sar & Summer Active Reading & Maxwell et al. (2014a \\
\hline text & TextNow & Maxwell et al. $2014 \mathrm{~b}$ \\
\hline tfl & Talk for Literacy & $\begin{array}{l}\text { Styles and Bradshaw } \\
(2015)\end{array}$ \\
\hline uos & Units of Sound & $\begin{array}{l}\text { Sheard, Chambers, } \\
\text { and Elliott }(2015) \\
\end{array}$ \\
\hline ve & Vocabulary Enrichment & Styles et al. (2014) \\
\hline catchl & Catch Up Literacy & Rutt $(2015$ \\
\hline $\mathrm{p} 4 \mathrm{~cm}, \mathrm{p} 4 \mathrm{cr}, \mathrm{p} 4 \mathrm{cw}$ & Philosophy for Children & $\begin{array}{l}\text { Gorard, Siddiqui, and } \\
\text { See }(2015 \mathrm{c})\end{array}$ \\
\hline pbcp & Perry Beeches Coaching Programme & Lord et al. 2015 \\
\hline pr7, pr9 & Paird Reading & Lloyd et al. $(2015)$ \\
\hline shine & SHINE in Secondaries & Menzies et al. $(\overline{2015})$ \\
\hline ttpe, ttpm, ttse, ttsm & Tutor Trust & Buchanan et al. \\
\hline aspl, aspm & Creative Futures: Act, Sing, Play & Haywood et al. (2015) \\
\hline impl, impn & Improving Numeracy and Literacy & $\begin{array}{l}\text { Worth, Sizmur, and } \\
\text { Styles }(2015)\end{array}$ \\
\hline quest & Quest & Biggart $(2015)$ \\
\hline wwr & Word and World Reading & $\begin{array}{l}\text { See, Gorard, and Sid- } \\
\text { diqui (2015) }\end{array}$ \\
\hline chess & Chess in Schools & Jerrim et al. $(2016)$ \\
\hline rflct & ReflectED & Motteram et al. \\
\hline
\end{tabular}

Table 1.: EEF project information. We abbreviate full EEF titles to labels that mark each of the 50 outcomes examined. The references to full evaluation reports are also listed. 